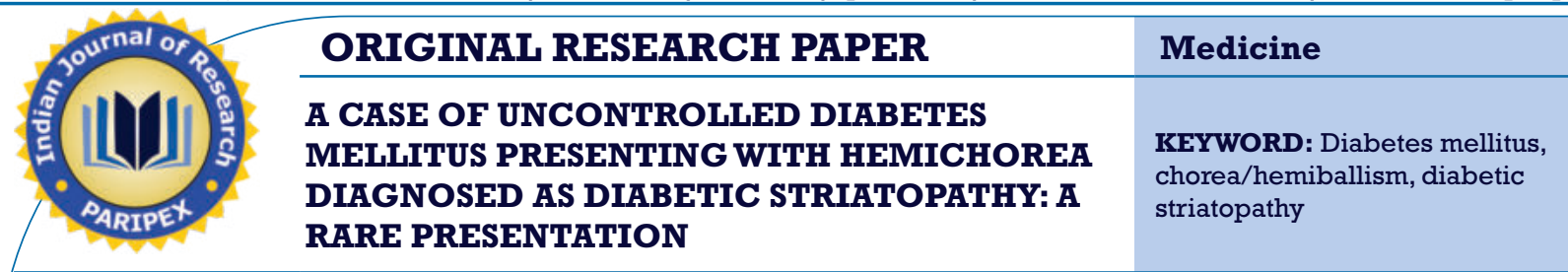

\section{Sumanta Sinha}

\section{Rupak Chatterjee}

Kumkum

Sarkar*

Debananda Gonjhu

\section{Sekhar Pal}

\section{Netai Pramanik}

M. B. B. S., DTM\&H, MD PGT, Dept. of Tropical Medicine, School of Tropical Medicine, Kolkata.

M. B. B. S., MD PGT, Dept. of Tropical Medicine, School of Tropical Medicine, Kolkata.

M. B. B. S., MD, Assistant Professor, Dept. of Tropical Medicine, School of Tropical Medicine, Kolkata. *Corresponding Author

M. B. B. S., MD, Assistant Professor, Dept. of Tropical Medicine, School of Tropical Medicine, Kolkata.

M. B. B. S., MD, Assistant Professor, Dept. of Tropical Medicine, School of Tropical Medicine, Kolkata.

M. B. B. S.,DTM\&H, MD, Associate Professor, Dept. of Tropical Medicine, School of Tropical Medicine, Kolkata.

E We are all aware of the macrovascular and microvascular complications of diabetes mellitus but till date diabetic

striatopathy has been under reported as a complication of uncontrolled diabetes in patients presenting with hemichorea/hemi ballism. We are presenting a case of hemichorea in a case of uncontrolled diabetes mellitus as it is a rare association.

\section{INTRODUCTION:}

Diabetic Striatopathy (DS) has been defined as a hyperglycaemic condition associated with both or either one of the two following conditions: (1) chorea/ballism; (2) striatal hyperdensity on CT or hyperintensity on Tl-weighted MRI brain (1)(2)(3). Apart from DS, other probable causes of chorea/ballism may broadly be classified into causes due to any cerebrovascular, autoimmune, toxic, malignant, and infectious illness causes (4). The etiopathogenesis of chorea/ballism is attributed largely to the dysfunction of basal ganglia and subthalamus (5).

\section{CASE:}

A 58 year old diabetic and hypertensive male presented to us with sudden onset jerky and writhing involuntary movement over both left upper and lower limb for last 16-18 days. This involuntary movement was sudden in onset was slowly progressive during first 4 to 5 days. It was not associated with blurring of vision, altered mentation, nausea, vomiting, headache, loss of consciousness, slurring of speech, limb weakness, incontinence of stool or urine palpitation, chest pain, shortness of breath, fever, cough, loose stool, pain abdomen, rash, recent change in drug regime, recent travel, difficulty in deglutition, drooling of saliva, joint pain, difficulty in standing from sitting or sitting from standing posture. No history suggestive of hair loss, oral ulceration, tightening of skin, malar rash, exertional dyspnoea. No history of fleeting joint pain, recurrent throat infection. He had a history of very high blood glucose instead of taking multiple oral drugs.

His general and systemic examinations were normal but CBG was $587 \mathrm{mg} / \mathrm{dl}$ on admission. The patient was conservatively managed. Diabetic ketoacidosis was ruled out. Patient was put on diabetic salt restricted diet, basal bolus insulin regime in the form of regular and glargine insulin combination with oral hypoglycaemics in the form of metformin and linagliptin as sensitizer, oral antihypertensives in the form of amlodipine and telmisartan, antidyslipidemics in the form of atorvastatin and fenofibrate. Tab.Risperidone was added after consultation with the Dept. of Neurology. His routine blood examinations were normal but Brain MRI showed mildly hyperintense signals from the right caudate, adjacent putamen and left thalamic region showing enhancement in post contrast study which could be due to metabolic causes with special reference to ketoacidosis along with generalised cerebral and cerebellar atrophy.

On subsequent treatment patient gradually improved in his glycaemic control along with gradual decrease and ultimate eradication of the involuntary movement during treatment in the hospital for twelve days. On $12^{\text {th }}$ day patient was discharged with well controlled blood glucose level with no residual involuntary movement or any neurological deficit with normal vitals.

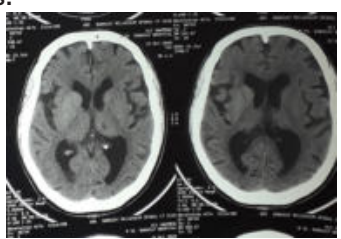

CT Shows Subtle Hyper Attenuation In The Right Putamen

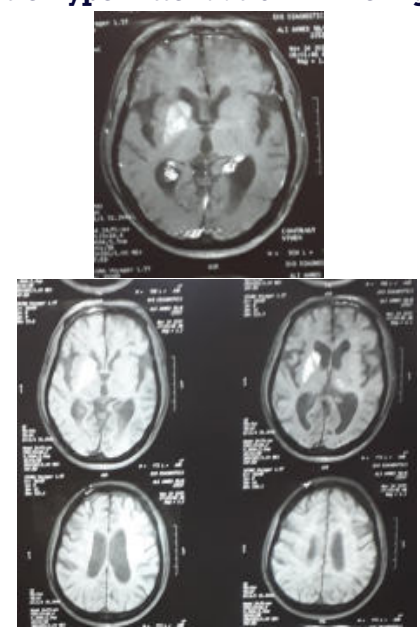

SIGNAL INTENSITY NOTED IN THE RIGHT PUTAMEN AND CAUDATE NUCLEAUS IN T1 WEIGHTED MRI BRAIN 


\section{IMA GE. SPARING OFTHE INTERNAL CAPSULE}

\section{DISCUSSION}

Studies have shown that although majority of DS presented with chorea/ hemichorea, there were few cases patients who presented with altered mental status (3)(6), convulsion (6), paresis (7), dysphagia and dysarthria (7).

Regarding the association between DS and ketosis, studies show that majority of patients of DS are not ketotic, compatible with the term "hyperglycaemic non-ketotic hemichorea/ hemi ballism". On the other hand, ketosis in the remaining patients implied that occurrence of DS is not exclusively restricted to non-ketotic patients. (8). In our case we found the patient to be non-ketotic.

The preponderance of DS attributable to non-ketotic hyperglycaemic condition may arise from the underlying pathophysiology of choreo-athetoid movement. In nonketotic hyperglycaemic status, brain metabolism mechanism converts to the alternative anaerobic pathway in Krebs cycle that results in rapid depletion of gamma-aminobutyric acid ( $G A B A)$ leading to disinhibition of subthalamus and basal ganglia grossly responsible for hyperkinetic movements in DS patients. On the other hand, in ketosis, GABA can be regenerated by using acetoacetate synthesised in the liver to prevent its reduction, thereby explaining the rarity of DS in diabetic ketoacidosis (4)(2)

As per previous case reports (4), most common pattern of striatal abnormalities of DS has been identified as isolated putamen involvement, followed by combined caudate nucleus-putamen involvement. Anomalies involving all three striatal components has been found in some of the cases.

CT and MRI are the two commonest imaging modalities to detect striatal anomalies of DS.

Although multiple mechanisms and multiple aetiologies may result in striatal hyperintensity on Tl-weighted MRI brain imaging including hypertensive bleed, genetic diseases (e.g., Tay-Sachs disease, tuberous sclerosis, neurofibromatosis),calcification, metabolic disorders (e.g., Wilson's disease, hypoglycaemic coma, chronic hepatic encephalopathy), toxicity (e.g., manganese toxicity, carbon monoxide poisoning), and brain ischemia (e.g. postcardiac arrest encephalopathy), the striatal lesions are mostly bilateral except in intracranial haemorrhage secondary to hypertension(9). One of the significant and salient features of DS-associated striatal lesion to differentiate it from hypertensive bleed is the absence of mass effect and the sparing of the internal capsule (10). This unique finding, when combined with hyperglycaemia and the presence of chorea is the DS.These findings fit to our case.

The mainstay of DS treatment is control of hyperglycaemia with proper hydration to correct the underlying metabolic abnormality (2). Several studies have concluded that although chorea can be successfully treated with implementation of adequate glycaemic control only in a proportion of the patients, the majority required additional anti-chorea medications for alleviation of symptom complex. Four main categories of anti-chorea medications are prescribed for the purpose namely antipsychotics, GABAreceptor agonists, selective serotonin reuptake inhibitors and dopamine-depleting agents (2). Several studies showed that haloperidol is the most common agent against DS-associated chorea, followed by tetrabenazine, risperidone and clonazepam. Other medications included valproate, tiapride, quetiapine, pimozide, diazepam etc.

We are presenting this case as we still find that apart from the macrovascular and microvascular complications of diabetes mellitus diabetic striatopathy is an under reported finding till date.

www.worldwidejournals.com

\section{REFERENCES:}

1. Abe, Y. et al. Diabetic striatal disease: clinical presentation, neuroimaging, and pathology. Intern. Med.48, 1135-1141 (2009).

2. Das, L., Pal, R., Dutta, P.\& Bhansali, A. "Diabetic striatopathy" and ketoacidosis: Report of two cases and review of literature. Diabetes Res. Clin. Pract. 128, 1-5 (2017).

3. Sato, H. et al. Diabetic striatopathy manifesting as severe consciousness disturbance with no involuntary movements. Diabet. Med. 34, 1795-1799 (2017).

4. Oh, S.-H., Lee, K.-Y., Im, J.-H. \& Lee, M.-S. Chorea associated with non-ketotic hyperglycemia and hyperintensity basal ganglia lesion on Tl-weighted brain MRI study: a meta-analysis of 53 cases including four present cases. J. Neurol.Sci.200,57-62 (2002).

5. Ohmori, H. et al. Two cases of hemiballism-hemichorea with Tl-weighted MR image hyperintensities. Intern. Med. 44, 1280-1285 (2005).

6. Shobha, N., Sinha, S., Taly, A., Pal, P. \& Chandrasekhar, H. Diabetic nonketotic hyperosmolar state: Interesting imaging observations in 2 patients with involuntary movements and seizures. Neurol. India 54,440-442 (2006).

7. Tung, C.-S., Guo, Y.-C., Lai, C.-L. \& Liou, L.-M. Irreversible striatal neuroimaging abnormalities secondary to prolonged, uncontrolled diabetes mellitus in the setting of progressive focal neurological symptoms. Neurol. Sci.31,57-60 (2010)

8. Choon-Bing Chua,Cheuk-Kwan Sun, Chih-Wei Hsu, Yi-Cheng Tai, Chih-Yu Liang \& I-Ting Tsai. "Diabetic striatopathy": clinical presentations, controversy, pathogenesis, treatments, and outcomes. www.nature.com/ scientificreports. (2020) 10:1594 | https:// doi.org/ 10.1038/ s41598- 02058555-w

9. Suárez-Vega, V. M. et al. CT and MR unilateral brain features secondary to nonketotic hyperglycemia presenting as hemichoreahemiballism. Case Rep Radiol 2016 (2016)

10. Sitburana, O. \& Ondo, W. G. Tetrabenazine for hyperglycemic-induced hemichorea-hemiballismus. Mov.Disord.21,2023-2025 (2006). 